\title{
Diamond/Cu Composites Fabricated by Pressureless Infiltration Method
}

\author{
Jia Jin-Hao ", Xiong De-Gan, Bai Shu-Xin, Guo Wei-Qiang, Du Guang-Bao \\ College of Aerospace Science and Engineering, National University of Defense Technology, Changsha, China
}

\section{Email address:}

jiajinhao12@foxmail.com (Jia Jin-Hao)

${ }^{*}$ Corresponding author

\section{To cite this article:}

Jia Jin-Hao, Xiong De-Gan, Bai Shu-Xin, Guo Wei-Qiang, Du Guang-Bao. Diamond/Cu Composites Fabricated by Pressureless Infiltration Method. Science Discovery. Vol. 4, No. 2, 2016, pp. 151-155. doi: 10.11648/j.sd.20160402.26

Received: April 26, 2016; Accepted: May 20, 2016; Published: May 23, 2016

\begin{abstract}
Copper matrix composites reinforced diamond (diamond/Cu) composites were successfully fabricated by pressureless infiltration method. The composition and structure of the coating are investigated using scanning electron microscopy(SEM), X-ray diffraction. The results indicate that the composition of the coatings is WC which is uniform on diamond. The microstructure and properties of diamond/Cu composites were investigated. The results show that the diamond/Cu composites were compact and WC transition layers onto diamond can greatly promote the compatibility between diamond and $\mathrm{Cu}$. Further investigation reveals that the diamond $/ \mathrm{Cu}$ composites possess low density $\left(5.3907 \mathrm{~g} / \mathrm{cm}^{3}\right)$, excellent thermal management function as a result of high thermal conductivity up to $761.1 \mathrm{~W} /(\mathrm{m} \cdot \mathrm{K})$ and a fine tightness $\left(2.5 \times 10^{-10} \mathrm{~Pa} \bullet \mathrm{m}^{3} / \mathrm{s}\right)$.
\end{abstract}

Keywords: Pressureless Infiltration Method, Diamond/Cu Composites, Coating

\section{无压浸渗法制备Diamond/Cu复合材料}

贾进浩*, 熊德赣, 白书欣, 郭伟沯, 杜广报

航天科学与工程学院, 国防科学技术大学, 长沙, 中国

\section{邮箱}

jiajinhao12@foxmail.com（贾进浩）

中文摘要: 用无压浸渗法成功制备了金刚石颗粒增强铜基体 (Diamond $/ \mathrm{Cu}$ ) 复合材料。用扫描电镜和X衍射仪对金刚石 表面涂层进行表征, 发现金刚石颗粒表面的涂层均匀并且其相组成为WC。同时对, 金刚石铜复合材料的显微组织及性 能进行研究。结果表明, Diamond/Cu复合材料致密, 并且发现碳化铇镀层显著改善了铜和金刚石之间的润湿性。进一 步研究发现Diamond/Cu复合材料具有低密度 $\left(5.3907 \mathrm{~g} / \mathrm{cm}^{3}\right)$ 、热导率高达 $761.1 \mathrm{~W} /(\mathrm{m} \bullet \mathrm{K})$ 和气密性好 $\left(2.5 \times 10^{-10} \mathrm{~Pa} \bullet \mathrm{m}^{3} / \mathrm{s}\right)$ 的特性。

关键词: 无压浸渗法, Diamond/Cu复合材料, 涂层

\section{1. 引言}

以SiC为代表的第三代半导体材料由于发光效率高、 击穿电场高、热导率高、电子饱和速率高及抗辐射能力强,
特别适于制作高温、高频、抗辐射及大功率器件。这对封 装技术、封装材料提出了严峻的挑战 $[1-3]$ 。

金刚石铜复合材料以下简写为 (Diamond $/ \mathrm{Cu}$ ) 具有导 热率高 $(\geqslant 600 \mathrm{~W} /(\mathrm{m} \bullet \mathrm{K}))$ 、热膨胀系数较低 $\left(5 \sim 7 \times 10^{-6} / \mathrm{K}\right)$ 、 
密度小、净成可实现复杂形状的制备等优良特点有望取代 目前广泛应用的 $\mathrm{Cu} 、 \mathrm{Al} / \mathrm{SiC} 、 \mathrm{Al} 、 \mathrm{~W} / \mathrm{Cu} 、 \mathrm{Mo} / \mathrm{Cu} 、 \mathrm{Be} 0$ 等 材料。但是金刚石和铜间附着功仅为 $316 \times 10^{-7}$ 、润湿角在 $1100^{\circ} \mathrm{C}$ 真空时为 $140^{\circ}$, 所以两相的界面结合强度低, 很难 制备得到高致密，高热导的Diamond/Cu复合材料 [4]。为 了改善金刚石与铜基体的界面结合状况，常用的方法有： 金刚石表面金属化即通过过渡族元素和周期表中第 IV 周 期元素, 如W, Mo, Cr, Ti 等与金刚石表面反应形成碳化物改 善与基体铜的润湿性; 铜基体添加合金元素（B, Cr, Ti, V, 稀土）以降低液态铜的表面张力; 压力控制如高温高压技 术来改善界面结合状况 [5]。目前制备Diamond $/ \mathrm{Cu}$ 复合材 料的方法有粉末冶金、热压烧结、放电等离子烧结、压力 熔渗、高温高压等方法，但是这些方法制备Diamond $/ \mathrm{Cu}$ 复合材料成本高、工艺复杂，难以有效降低生产成本，无 压浸渗法制备Diamond/Cu复合材料由于设备要求及工艺 参数简单可以实现大规模生产从而有效降低成本 [6-9].

WC的热导率较高 $(147 \mathrm{~W} /(\mathrm{m} \bullet \mathrm{K}))$ 热膨胀系数小 $(3.9$ $\left.\times 10^{-6} / \mathrm{K}\right)$ 与金刚石颗粒的热匹配好, 可以有效改善金刚石 与铜液的润湿性、降低界面热阻。基于上述特性本实验的 目的在于探索金刚石表面磁控溅射镀铇与无压浸渗法制 备Diamond/Cu复合材料的可行性。同时也研究金刚石表面 磁控溅射铇热处理后的表面组织及Diamond $/ \mathrm{Cu}$ 复合材料 的微观组织和物理特性 [10]

\section{2. 试验}

\section{1. 原材料}

实验所用金刚石颗粒为商业用MBD-4级粒径为 $80 / 100$ 目。所用铜为无氧铜, 纯度为 $99.995 \%$ 密度为 $8.94 \mathrm{~g} / \mathrm{cm}^{3}$ 。 物理特性如表1所示。

表1 原材料的物理性能。

\begin{tabular}{llll}
\hline materials & $\mathrm{CTE} / \mathrm{ppm} /{ }^{\circ} \mathrm{C}$ & $\mathrm{TC} / \mathrm{W} /(\mathrm{m} \cdot \mathrm{K})$ & Density $/\left(\mathrm{g} / \mathrm{cm}^{3}\right)$ \\
\hline $\mathrm{Cu}$ & 17.0 & 400 & 8.94 \\
Diamond & 3.73 & 1350 & 3.52 \\
\hline
\end{tabular}

\section{2. 实验过程}

图1是本论文的实验流程图。



图1 实验流程图。

金刚石颗粒表面预处理: 首先将金刚石颗粒置于丙酮 液中超声 15 分钟, 再依次用酒精、去离子水清洗数次后置 于 $10 \%$ 的氢氧化钠溶液中煮沸 10 分钟, 取出后用去离子水
清洗至中性，烘干金刚石颗粒后将其置于 $30 \%$ 的稀硝酸中 煮沸25分钟，后去离子水冲洗至中性。金刚石表面磁控溅 射铇 $9 \mathrm{~h}$, 并将镀覆有铇的金刚石粉真空高温 $\left(1050^{\circ} \mathrm{C} \times\right.$ $2 \mathrm{~h}, 10 \mathrm{~Pa}$ ) 处理, 模具固定金刚石颗粒, 放置铜块于模具中。 最后将其放到真空炉中, 炉内压力保持在 $2.5 \mathrm{~Pa}$ 左右, 在 $1300^{\circ} \mathrm{C}$ 保温 $45 \mathrm{~min}$, 随炉冷却至室温, 待到温度降到 $500^{\circ} \mathrm{C}$ 卸压。制备所得Diamond/Cu复合材料的尺寸为 $\Phi 12.7 \mathrm{~mm}$ $\mathrm{x} 4 \mathrm{~mm}$ 。如图2所示。



图2 无压浸渗法制备Diamond/Cu复合材料的宏观形貌。

\section{3. 性能测试方法}

M6700场发射扫描电镜观察复合材料断面形貌, EDS 能谱分析界面的成分。德国耐驰LFA447型激光闪射仪法测 定复合材料的热扩散系数。材料的气密性用ZQJ-542 (KYKY Corp.) 氦质谱仪检测。用型号为ICT-3450高分辨 CT验证材料的致密性。用Archimedes排水法测量复合材料 的密度。采用D8 Advance X射线衍射仪对金刚石颗粒表面 镀层进行物相分析。试样尺寸为 $12.7 \mathrm{~mm} \mathrm{x} 4 \mathrm{~mm}$ 。

\section{3. 结果与分析}

Diamond $/ \mathrm{Cu}$ 复合材料的性能如表1所示, 测试结果表 明材料具有密度低, 气密性好, 热导率高的优异的特性。 能满足现代电子封装器件对材料性能的要求。

表2. 制备所得Diamond/Cu复合材料的性质。

\begin{tabular}{llllll}
\hline materials & Volume ration of diamond\% & Gas tightness $/\left(\mathrm{mPa} \cdot \mathrm{cm}^{3} / \mathrm{s}^{1}\right)$ & Density $/\left(\mathrm{g} / \mathrm{cm}^{3}\right)$ & Thermal diffusivity $\left(\mathrm{m}^{2} / \mathrm{s}\right)$ & $\mathrm{TC} / \mathrm{W} /(\mathrm{m} \bullet \mathrm{K})$ \\
\hline Diamond $/ \mathrm{Cu}$ & $65.48 \%$ & $2.5 \times 10^{-10}$ & 5.3907 & 328.348 & 761.1 \\
\hline
\end{tabular}




\section{1. 镀铇金刚石颗粒高温处理的结果}

金刚石颗粒镀铇后高温处理后的形貌如图3所示。从 图中可以看出, 镀层完整性较好, 镀层的不连续主要分布 在金刚石颗粒的棱角处。图 4 是利用 X-ray衍射技术对镀层 的物相进行分析得到的谱图。可知主要物相为WC。



图3 镀铇金刚石颗粒高温处理后金刚石形貌 $(\mathrm{SEM}) \times 150$ 。



图4 镀覆金刚石的X射线衍射图。

\section{2. Diamond $/ \mathrm{Cu}$ 微观组织观察}

通过断口形貌可以更加直观地观察和分析复合材料 界面形貌以及两相结合情况. 图5是无压浸渗制备得到的 $\mathrm{diamond} / \mathrm{Cu}$ 复合材料典型断口的SEM照片。由图5可以看到 Diamond $/ \mathrm{Cu}$ 复合材料的组织致密, 颗粒分布均匀。同时 Diamond颗粒棱角分明, 很好地维持了最初始的外形, 均 匀地镶嵌在铜基体中, 无颗粒团聚和搭接现象。这主要得 益于金刚石颗粒间孔隙的连通性及金刚石表面金属化改 善了液一固两相的润湿，使得基体充分填充到颗粒的间隙 中。基体金属沿金刚石与铜连接处的沿晶断裂, 从图5(b) 中还可看出金刚石表面呈较光洁状态, 且多个位置的金刚 石已从铜基体中拔出, 说明界面结合弱于金刚石本身, 同 时也说明金刚石表面金属化后与基体之间是不连续的机 械咬合，从图7也可以得到证明。这是由于界面处两相既 不发生反应又不发生扩散, 所以界面结合力为强度较低机
械咬合。从图5 (b) 中发现材料内部有凹陷处有少的孔隙, 结合气密性数据得知这是金刚石脱落所形成的。从图 5 (c) 中可以观察到铜铺展包覆在了镀铇金刚石表面, 说明镀铇 金刚石颗粒经高温处理后和铜液之间的润湿效果很好。在 近垂直于断口方向的界面位置有基体金属与金刚石颗粒 的两相剥离, 各颗粒表面的残留金属呈不连续分布以上断 口形貌特征指明, 在复合材料断裂过程中, 基体铜发生塑 形破坏，而两相界面可以稳定存在


图5 Diamond/Cu复合材料的断面形貌。 


\section{3. Diamond/Cu复合材料界面分析}

材料断口的扫描电镜组织分析和EDS能谱分析结果见 图6和图7。从上图6中可以观察到一部分金刚石金属化后 表面镀层存在明显的破裂。这是样件被破坏时剥落的。在 不考虑基体铜的影响下从图 $\mathrm{a}$ 的先扫描结果可以看出祄度 较深的部分显示的是金刚石颗粒表面即能谱结果为碳。而 祄度较浅的部分从能谱结果蓝色的曲线和红色的曲线可 以看出是铇和碳。再结合上图4所示结果可知金刚石表面 祄度较浅的一层为WC铜包覆于其上。即Diamond $/ \mathrm{Cu}$ 复合材 料界面如图7所示。结合图6和图7可知镀覆金刚石颗粒之 间形成了Diamond -WC-Cu-WC-Diamond的过渡。如界面示 意图8所示。Diamond $/ \mathrm{Cu}$ 复合材料的热导率为 $761 \mathrm{~W} /(\mathrm{m} \bullet \mathrm{K})$, 这有赖于界面的结合状态。机械结合的强弱很大程度上依 赖于基体表面的粗粘度, 因此适当地提高基体表面的粗糙 度将促进镀层与基体结合力的提高 [11]。


图6 Diamond/Cu复合材料金刚石表面形貌a), b) 线扫图。


图7 Diamond/Cu复合材料界面形貌a), b) 线扫图。

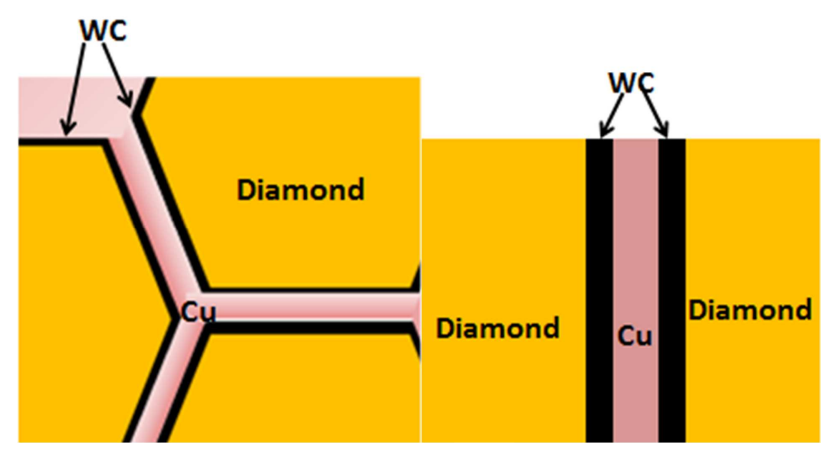

图8 Diamond/Cu复合材料界面结构示意图。

\section{4. Diamond/Cu复合材料的气密性}

测得Diamond $/ \mathrm{Cu}$ 复合材料和参比铜块的气密性数据 列于表 3 。从两个数据的比较中得出Diamond $/ \mathrm{Cu}$ 复合材料 的致密性很好, 这说明实验的熔渗温度、压力、镀层和金 刚石颗粒尺寸很适合制备高导热和高致密的Diamond $/ \mathrm{Cu}$ 材料。同时也可以推断出所制备得到的Diamond/Cu复合材 料中金刚石颗粒间的搭接和缺陷非常少。说明铜液能顺利 的渗入表面已经金属化金刚石颗粒之间, 从侧面也说明金 刚石表面金属化提高与铜液的润湿性。同时从图9是高分 辨率CT扫描图可以看出材料均匀致密, 无明显的缺陷。

表3 制备所得Diamond $/ \mathrm{Cu}$ 气密性和铜块比较.

\begin{tabular}{lll}
\hline materials & Mass ration of $\mathrm{Cu} \%$ & Gas tightness $\left(\mathrm{mPa} \cdot \mathrm{cm}^{3} / \mathrm{s}^{1}\right)$ \\
\hline $\mathrm{Cu}$ & 99.995 & $1.3 \times 10^{-10}$ \\
Diamond $/ \mathrm{Cu}$ & 57 & $2.5 \times 10^{-10}$ \\
\hline
\end{tabular}



图9 Diamond/Cu复合材料 $\mathrm{CT}$ 扫描图。

\section{4. 结论}

1. 镀铇金刚石颗粒真空高温 $\left(1050^{\circ} \mathrm{C} \times 2 \mathrm{~h}\right)$ 处理后其表 面生成的WC有效改善了金刚石与铜液的润湿。

2. 采用金刚石表面改性和无压浸渗的工艺成功制备出 了热导率高达 $761.1 \mathrm{~W} /(\mathrm{m} \bullet \mathrm{K})$ 。气密性为 $2.5 \times 10^{-10}$ $\mathrm{mPa} \cdot \mathrm{cm}^{3} / \mathrm{s}^{1}$ 的Diamond $/ \mathrm{Cu}$ (65.48\% Diamond) 的复合材 料。

3. Diamond/Cu复合材料具有致密、均匀的微观组织结 构, 其断裂特征为基体的韧性断裂。

\section{参考文献}

１1］高文珈，贾成光，楮克. 金刚石/金属基复合新型热管理材 料的研究与进展 [J]. 材料导报, 2011，25（2）：17-26。

[2] 李 信, 龙剑平, 胥明. 金刚石颗粒/金属基复合材料的研 究进展 $[J]$. 特种铸造及有色合金, $2012 ， 32 （ 7 ）$ ：654656 。 
[3] Zweben C., High-performance thermal management materials $[J]$. Advanced packaging, 2006, 15: 20。

[4] CHEN Chao, GU0 Hong. Thermal conductivity of diamond/copper composites with a bimodal distribution of diamond particle sizes prepared by pressure infiltration method [J]. RARE METALS, 2011, $30(4): 408$.

[5] XIA Yang. Influence of Interfaces on Thermal Conductivity of Diamond-Cu Composites for Heat Sink Application [J]. JOURNAL OF SYNTHETIC CRYSTALS, 2009, 38(1) : 171-174.

[6] Andrey M. Abyzov• Sergey V. Kidalov •Fedor M. Shakhov. High thermal conductivity composites consisting of diamond fillerwith tungsten coating and copper (silver) matrix [J]. Mater Sci, 2011, 46: 1424-1438.

[7] William B. Johnsonand B. Sonuparlak. Diamond/Al metal matrix composites formed by the pressureless metal infiltration process [J]. Mater. Res, 1993, 8(5): 1171-1173。
[8] MA Shuangya. Studyon Thermal Conductivity of Diamond / Copper Composite [J]. Material\&Heat Treatment, 2008, 37: 36-38.

[9] Congxu Zhu, Chan Wang, Nangang Maet. Si-coated diamond particles reinforced copper composites fabricated by spark plasma sintering process [J]. Materials and Manufacturing Processes. 2013, 28: 143-147.

[10] Chun Zhang • Richu Wang $\bullet$ Zhiyong Cai. Low-temperature densification of diamond/Cu compositeprepared from dual-layer coated diamond particles [J]. Mater Electron, 2015, 26: $185-190$.

[11] Hua Bai, Nangang Ma, Jing Lang, Ying Jin, Congxu Zhu, Yi Ma. Thermo-physical properties of boron carbide reinforced copper composites fabricated by electroless deposition process [J]. Mater Des. 2013, 46: $740-74$. 